\begin{tabular}{|c|c|}
\hline Title & $\begin{array}{l}\text { Persistent High Polarization of Excited Spin Ensembles During Light Emission in Semiconductor Quantum-Dot-Well } \\
\text { Hybrid Nanosystems }\end{array}$ \\
\hline Author(s) & $\begin{array}{l}\text { Takeishi, Kazuki; Hiura, Satoshi; Takayama, Junichi; Itabashi, Kodai; Urabe, Masay uki; W ashida, A kihiro; Kiba, } \\
\text { T akayuki; Murayama, A kihiro }\end{array}$ \\
\hline Citation & $\begin{array}{l}\text { Physical review applied, } 10(3), 034015 \\
\text { https://doi.org/10.1103/Phy sRevA pplied.10.034015 }\end{array}$ \\
\hline Issue Date & $2018-09-10$ \\
\hline Doc URL & http:/hdl.handle.net/2115/71643 \\
\hline Rights & @2018 A merican Physical Society \\
\hline Tyре & article \\
\hline Additional Information & There are other files related to this item in HUSCAP. Check the above URL. \\
\hline File Information & Phy sRevA pplied.10.034015.pdf \\
\hline
\end{tabular}

Instructions for use 


\title{
Persistent High Polarization of Excited Spin Ensembles During Light Emission in Semiconductor Quantum-Dot-Well Hybrid Nanosystems
}

\author{
Kazuki Takeishi, ${ }^{1}$ Satoshi Hiura, ${ }^{1}$ Junichi Takayama, ${ }^{1}$ Kodai Itabashi, ${ }^{1}$ Masayuki Urabe, ${ }^{1}$ \\ Akihiro Washida, ${ }^{1}$ Takayuki Kiba, ${ }^{2}$ and Akihiro Murayama ${ }^{1,{ }^{*}}$ \\ ${ }^{1}$ Graduate School of Information Science and Technology, Hokkaido University, Kita 14, Nishi 9, Kita-ku, \\ Sapporo 060-0814, Japan \\ ${ }^{2}$ Kitami Institute of Technology, 165 Koen-cho, Kitami 090-8507, Japan
}

(Received 5 March 2018; revised manuscript received 2 July 2018; published 10 September 2018)

\begin{abstract}
We demonstrate persistent high degrees of spin polarization (SPD) up to 70\% during light emission in $\left(\mathrm{In}_{1-x} \mathrm{Ga}_{x}\right)$ As quantum-dot-well (QD-QW) hybrid nanosystems, where QD excited states are laterally tunnel coupled through the adjacent two-dimensional QW potential depending on the QW thickness. Spinpolarized electrons are photo-excited by using circularly polarized light pulses. The decay time of spin relaxation, obtained by that of the SPD, is 70 times larger than the photoluminescence decay time. The temporally constant SPD is sustained by a selective transfer of minority spins among QDs after flipping from the majority spins, which is promoted by a moderate state filling of lower-energy spin sublevels in surrounding QDs. The spin transfer times are deduced as functions of the QW thickness and excited-spin density. These results can provide a precise control of lateral interdot spin-transfer dynamics and resultant suppression of spin relaxation in QD ensembles.
\end{abstract}

DOI: 10.1103/PhysRevApplied.10.034015

\section{INTRODUCTION}

Semiconductor quantum dots (QDs) are regarded as the most promising nanomaterials for extremely low-energy consumption optical and photonic devices [1-3]. Selfassembly grown high-density III-V compound semiconductor QDs such as InAs and $\left(\mathrm{In}_{1-x} \mathrm{Ga}_{x}\right)$ As achieved high performances, not affected by ambient temperature [4,5]. Recently, top-down nanotechnology has provided welldesigned high-density QDs of GaAs and $\left(\operatorname{In}_{1-x} \mathrm{Ga}_{x}\right) \mathrm{N}[6,7]$. In addition, the semiconductor QDs exhibit significant suppression of carrier- or exciton-spin relaxation originating from motional freezing due to strong three-dimensional (3D) quantum confinements [8-10]. Therefore, if this characteristic spin property in QDs can be integrated into device structures, one can provide a new concept of an optical device that transmits and receives spin information superimposed on light and photons [11-14]. This concept will enable a technology platform for integration of quantum optoelectronics in future information processing with ultra-low-energy consumption. For this purpose, it is essential to maintain the spin polarization during light emission.

Carrier migration among high-density QDs is commonly observed due to tunneling between neighboring dots $[15,16]$. The tunneling of spin-polarized carriers can

\footnotetext{
*murayama@ist.hokudai.ac.jp
}

affect the total spin polarization of the QD ensemble. It is usually challenging to study the effects of wavefunction coupling without changes of other structural parameters such as the size, spacing, strain, and density. Vertically coupled QDs with various spacer thicknesses were investigated to demonstrate the wavefunction coupling and related carrier dynamics [17-19]. However, the stacking of a self-assembled QD layer can also often affect structural properties including the growth-dependent interdot spacing in addition to the above factors. Moreover, it is highly challenging to investigate the spin dynamics in laterally coupled QD systems, where many QDs can collaboratively contribute to the spin dynamics. This concept can also pave the way for the dynamical formation of a so-called spin network.

In this study, we use $\mathrm{In}_{0.5} \mathrm{Ga}_{0.5}$ As QD layers tunnelcoupled with quantum wells (QWs) of $\mathrm{In}_{0.1} \mathrm{Ga}_{0.9} \mathrm{As}$ through GaAs barriers. We reveal that the electron wavefunctions localized in each QD can be laterally coupled when the two-dimensional-(2D)-QW potential is close to a thin tunneling barrier. This tunnel-coupled QD-QW hybrid nanosystem is of significance as electron spins are laterally transferred among QDs through spin-conserving tunneling, which can be precisely controlled only by a simple structural parameter, the QW thickness $t$. We had already established the crystal growth of such a tunnel-coupled QD-QW structure [20,21]. Our previous studies using the QD-QW structures revealed an efficient and ultrafast 
spin injection from the QW into QDs with injection time constants smaller than $10 \mathrm{ps}$ and spin-conservation rates higher than $90 \%$. This spin transfer dynamics was not affected by temperature at least up to $200 \mathrm{~K}$; therefore, it is of importance for room-temperature device operation [22].

In addition, the spin-injection efficiency can be largely affected by the filling effect for spin-split states in QDs, where the spin polarization in QDs after injection degrades by the filling at majority spin states (Pauli's spin blocking). Minority spins can be continuously injected, while the injection of majority spins is blocked by the filling, which makes the number ratio of both spins closer leading to spin relaxation [23]. High-density QDs were used to overcome the spin blocking [24], which completely agrees with the development of QD lasers $[25,26]$. Spin-dependent filling can also affect the spin dynamics in the laterally coupled QD systems we investigate in this study.

Circularly polarized time-resolved PL is measured; the time-dependent circular polarization can directly reflect the time-dependent spin polarization at emissive states due to the optical selection rule [27]. QD ensembles with thicker QWs, where the interdot coupling is stronger, show features of a collective behavior of spin relaxation. We observe that the decay time of the circular polarization becomes 35 times larger than that of the PL intensity, which implies that the spin relaxation time becomes 70 times larger than the PL decay time. The mechanism is quantitatively discussed based on a rate-equation analysis and an effective rate of the spin transfer among QDs is determined as a function of the excited spin density.

\section{EXPERIMENTAL PROCEDURES}

The QD-QW-coupled structures are grown by molecular beam epitaxy; the details are described in previous studies [20-22]. Briefly, 400-nm-thick GaAs buffers are grown on GaAs (001) substrates at $580^{\circ} \mathrm{C}$. $\mathrm{In}_{0.1} \mathrm{Ga}_{0.9} \mathrm{As}$ QWs with thicknesses of $t=6,10$, and $20 \mathrm{~nm}$, and subsequently 8-nm-thick GaAs tunneling barriers are grown at $520^{\circ} \mathrm{C}$. The substrate temperature is decreased down to $500^{\circ} \mathrm{C}$ for a self-assembled growth of $\operatorname{In}_{0.5} \mathrm{Ga}_{0.5} \mathrm{As}$ QD layers with a nominal thickness of $2 \mathrm{~nm}$. The QD layers are immediately capped by a 10 -nm-thick GaAs layer after the QD formation, and subsequently by a $30-\mathrm{nm}$-thick GaAs layer at an increased temperature of $580^{\circ} \mathrm{C}$. The sheet density of the QD layer is approximately $2 \times 10^{10} \mathrm{~cm}^{-2}$, which is relatively high; however, it is designed so that the interdot coupling can be neglected. The average diameter is approximately $25 \mathrm{~nm}$. Circularly polarized light pulses of a mode-locked Ti:sapphire laser are employed as an excitation light source to perform time-synchronized electron-spin orientation in the GaAs barriers, where the light energy is tuned to $1.55 \mathrm{eV}$ to excite the bottom of the energy band of GaAs. The ideal initial circularpolarization degree (CPD) generated by this optical spin orientation is $50 \%$ at the GaAs barrier [27]. Time-resolved PL from the QDs and QWs is measured at $6 \mathrm{~K}$ using a streak camera. The PL helicity is discriminated using a pair of linear polarizer and quarter-wave plates.

\section{RESULTS AND DISCUSSION}

Figures 1(a) and 1(b) show a typical AFM image of the surface of the $\operatorname{In}_{0.5} \mathrm{Ga}_{0.5}$ As-QD layer grown at identical conditions and the 2D fast Fourier transform (2D FFT) image obtained from the AFM image. In Fig. 1(a), the AFM image shows closer dot separation along the $x$ direction than that along the $y$ direction, resulting from atomically-scaled steps on the substrate surface. The averaged nearest-neighbor distance of the horizontally aligned QDs in Fig. 1(a) is deduced to be $64 \mathrm{~nm}$ from the 2D FFT, as shown in Fig. 1(b). Figure 1(c) shows a high-angle annular-dark-field (HAADF) scanning transmission electron microscopy (STEM) image of the $\operatorname{In}_{0.5} \mathrm{Ga}_{0.5}$ As QD in the QD-QW coupled structure with a barrier thickness of $6 \mathrm{~nm}$ and QW thickness of $20 \mathrm{~nm}$, showing the single crystal QD structure with a diameter and height of 20 and $4 \mathrm{~nm}$, respectively. Figure 1(d) shows a TEM image of the whole QD-QW hybrid nanostructure, where the structure of this thinner barrier is observed as intentionally designed. Bright-field STEM shows local strain generation as dark contrast areas less than a few tens of nanometers in $z$ contrast of the QW as well as a more obvious strain generated in the QD area. However, strain is not observed in the GaAs barrier between the QW and QD layers, which is similar to the buffer layer below the QW. Therefore, we judge that strain generation in the GaAs barrier on the QW can be negligible, suggesting that the dot-growth conditions on this GaAs barrier layer are not significantly affected by the existence of QW and therefore, are almost identical among our samples.

The carrier wavefunctions in the QD-QW hybrid nanostructures are calculated using the NEXTNANO software [28]. The calculation models assume a 1-nm-thick wetting layer, two truncated pyramidal QDs with a base length of $20 \mathrm{~nm}$, and a center-to-center distance between the QDs of $60 \mathrm{~nm}$, considering the inner In concentration and resultant strain distributions, based on the above observations of the QD structures. Figures 1(g)-1(j) show spatial maps of the existence probability of the electron wavefunction at the first excited state (ES) of a QD as a function of $t$. The electron wavefunction without a QW is strongly localized at one of the two dots [Fig. 1(g)]. In contrast, the wavefunction spreads over the two neighboring dots in the samples with QWs [Figs. 1(h)-1(j)], and the degree of wavefunction penetration becomes more significant with the increase of $t$.

The calculations indicate that the existence of the adjusted 2D QW potential enhances the coupling strength of the electron wavefunctions among the laterally 

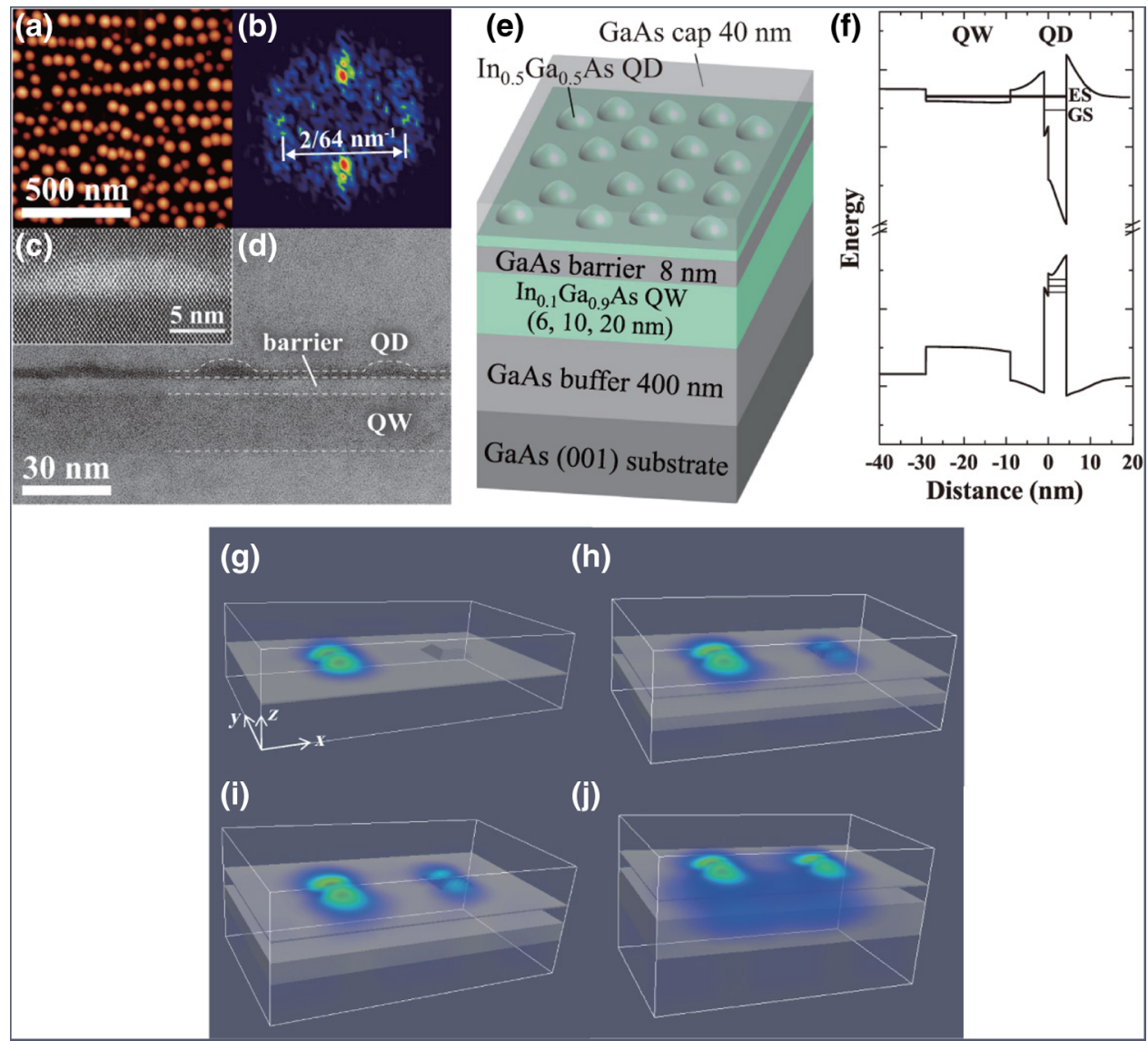

FIG. 1. (a) AFM image of the $\mathrm{In}_{0.5} \mathrm{Ga}_{0.5} \mathrm{As}$ QDs. (b) $2 \mathrm{D}$ FFT image from the AFM image in (a). (c) Cross-sectional HAADF-STEM lattice image of the $\mathrm{In}_{0.5} \mathrm{Ga}_{0.5} \mathrm{As}$ QD in the QD-QW hybrid nanostructure, where the degree of brightness reflects the In concentration. (d) Cross-sectional TEM image of the QD-QW hybrid nanostructure with a 6-nm-thick GaAs barrier and 20-nm-thick QW, where the dashed white lines are introduced to help the visualization. (e) Schematic drawing of the sample structure. (f) Schematic band alignment along the thickness direction and the resultant energy levels of an electron and a hole in the coupled QD-QW structure with a QW thickness of $20 \mathrm{~nm}$. 3D spatial maps of the existence probabilities of electron wavefunction at the first excited state (ES) between two neighboring QDs, (g) without a QW, and with (h) 6-nm-, (i) 10-nm-, and (j) 20-nm-thick QWs. aligned QDs. This implies that spin-polarized electrons migrate among QDs through the wavefunction coupling (tunneling) from higher-energy ESs to lower-energy ESs in surrounding QDs depending on the QD size and strain.

Figures 2(a)-2(c) show circularly polarized PL spectra and corresponding CPD from the QD-QW coupled structures with various $t$, where the GaAs barrier is excited using right-handed circularly polarized $\left(\sigma^{+}\right)$light pulses. $\mathrm{CPD}$ is defined as CPD $=\left(I_{\sigma+}-I_{\sigma-}\right) /\left(I_{\sigma+}+I_{\sigma-}\right)$, where $I_{\sigma+}$ and $I_{\sigma-}$ denote the PL intensities with co- and cross-circular polarizations for the excitation polarization, respectively. The broad PL spectra, from 1.3 to $1.4 \mathrm{eV}$, mainly correspond to ESs in the QD ensemble; the PL intensity decreases for lower energies due to the detection limit of the streak camera. PL spectra from the ground state (GS) are measured using a conventional CCD detector; the spectral peak energies are indicated by the black arrows in the figures. The weak but sharp PL spectral peak observed at the higher-energy region originates from the QW [2022]. A thicker QW sample exhibits a higher QW-PL intensity, accompanied by a shift of the PL peak toward lower energies and higher CPD. On the other hand, for the QDES spectrum, the CPD exhibits the maximum value around the higher-energy spectral edge and gradually decreases at lower energies. The QD samples with thicker QWs $(t=10$ and $20 \mathrm{~nm}$ ) exhibit the maximum CPD of $80 \%$, which is higher than that of the 6-nm-thick QW sample of 70\%. Time-response curves of these circularly polarized PLs from the QD ESs are shown in Figs. 2(d)-2(f). A single exponential decay curve of the time-dependent CPD is observed only in the sample with $t=6 \mathrm{~nm}$, while two components in the CPD decay curves are observed in the other samples. The sample with $t=6 \mathrm{~nm}$ exhibits a CPD decay time of $0.5 \mathrm{~ns}$, which is similar to that observed in the QD samples grown under identical conditions without QWs. The CPD decay curves of the samples with $t=10$ and $20 \mathrm{~nm}$ contain a similar decaying component in the time region larger than $0.4 \mathrm{~ns}$, where the PL intensities are almost negligible. In contrast, significantly slower decaying components in the CPD decay curves are observed in the time region smaller than $0.4 \mathrm{~ns}$, dominant during the PL emission. A surprisingly long, i.e., tentatively constant CPD decaying feature can be particularly observed in the sample with $t=20 \mathrm{~nm}$. From the analysis of the timeresolved PL spectra, we judge that the former PL CPD decaying component after $0.4 \mathrm{~ns}$ is caused by GSs with high energies confined in smaller dots in the QD ensemble. Therefore, we focus on the latter markedly slow CPD component originating only from ESs.

Figure 3 shows the time constants responsible for the circularly polarized PL decay curves as a function of $t$. The CPD decay time $\left(\tau_{\mathrm{CPD}}\right)$ corresponds to half of the time 


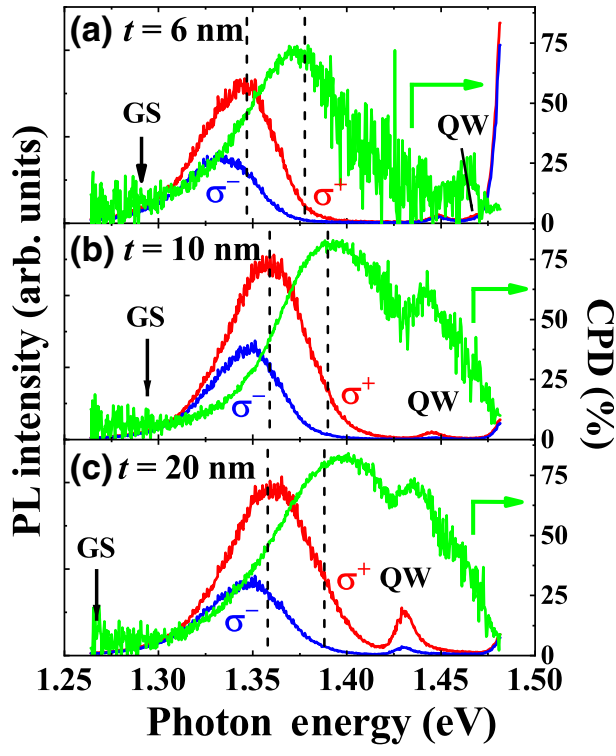

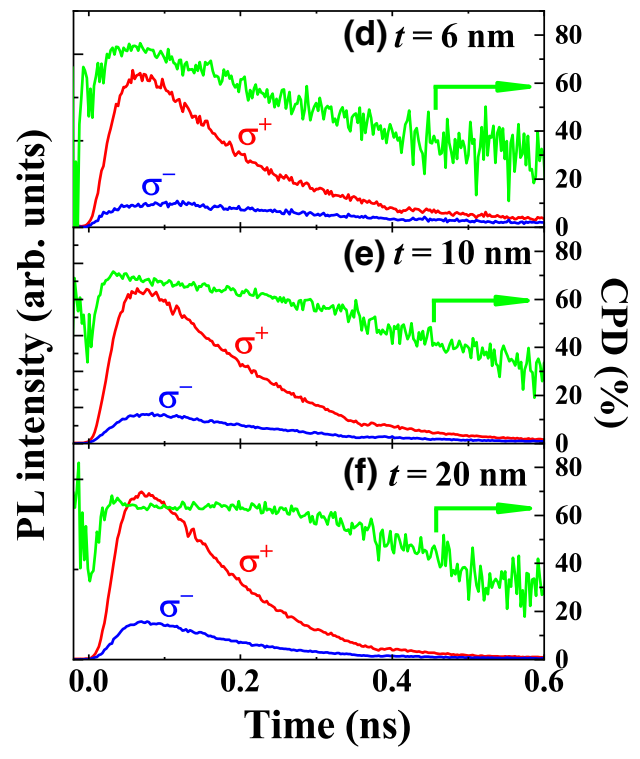

FIG. 2. Circularly polarized PL spectra and corresponding CPD as a function of the photon energy at $6 \mathrm{~K}$ from the QD-QW hybrid nanosystems with (a) 6-nm-, (b) 10-nm-, and (c) 20-nm-thick QWs at an excitation power of $2.5 \mathrm{~mW}$, where the spectral peak energy of the QD ground state (GS) is indicated by the black arrows. Circularly polarized transient PL and corresponding CPD as a function of the time in the samples with (d) 6-nm-, (e) 10-nm-, and (f) 20-nmthick QWs, measured at detection energy windows corresponding to the ESs in the QD ensembles, outlined by the black dashed lines in (a)-(c). constant of spin relaxation in a usual spin-split two-level system [29]. However, the CPD decay time in Fig. 3(a) systematically increases with $t$, and exhibits the maximum value of $3.6 \mathrm{~ns}$ for $t=20 \mathrm{~nm}$, corresponding to a spin relaxation time of $7.2 \mathrm{~ns}$. The ratio between the CPD decay time and the decay time of the PL intensity $\left(\tau_{\mathrm{PL}}\right)$ for the majority spin $\left(\sigma^{+}\right.$; co-polarization) is shown in Fig. 3(b). The maximum ratio reaches the value of 35 for $t=20 \mathrm{~nm}$, corresponding to 70 times higher value of the spin relaxation time compared with the PL decay time. This ratio is one order larger than that of about 6 to 7 observed in conventional QD samples without QWs [23]. In order to provide further insights, the decay times of the PL intensities for each polarization are plotted as a function of $t$ in Fig. 3(c). The difference in the PL decay times between the two polarizations is significant for $t=6 \mathrm{~nm}$, which reveals the CPD decaying characteristic as a function of time, i.e., the spin relaxation. However, this difference becomes smaller with the increase of $t$; the decay time of $\sigma^{-}$(cross-circular; minority spins) becomes smaller and the changes of $\sigma^{+}$(co-circular; majority spins) are significantly smaller. Therefore, the significant slowing of the CPD decay observed in the samples with thicker QWs can be directly attributed to the decrease of the PL decay time for the $\sigma^{-}$polarization (minority spins). The timeintegrated CPDs of the samples with $t=10$ and $20 \mathrm{~nm}$ exceed 80\%, as shown in Figs. 2(b) and 2(c).

Kalevich et al. reported a spin redistribution effect due to the Pauli blocking in QD ensembles [30]. Majority spins at ESs are restricted to relax to a lower-energy GS after the filling of the GS due to the spin-dependent Pauli blocking under a certain excitation power; only minority spins can relax to the GS. In this case, the spin polarization can be temporally enhanced at ESs after a pulsed spin excitation, i.e., the temporal PL CPD increases with the delay time up to the maximum, and then decreases as the spin population decreases due to emission. The maximum value of CPD is a function of the strength of the interdot coupling and excitation power, as shown using numerical calculations. According to the suggested model, we consider the spin transfer dynamics among the laterally tunnel-coupled QD ESs. The time-dependent spin dynamics are quantitatively analyzed using rate equations by considering the spin-state filling as well as spin relaxation [23].

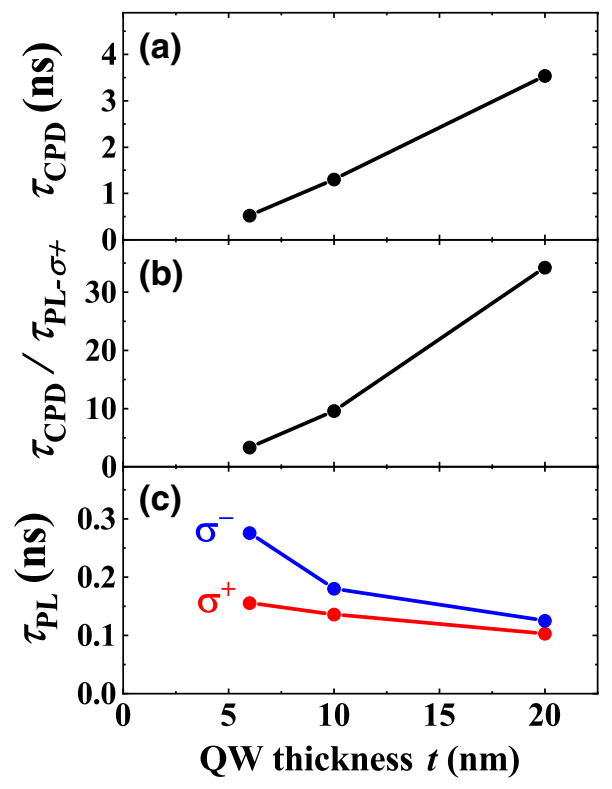

FIG. 3. Time constants (a) $\tau_{\mathrm{CPD}}$, (b) $\tau_{\mathrm{CPD}} / \tau_{\mathrm{PL}}^{\sigma+}$, and (c) $\tau_{\mathrm{PL}}^{\sigma \pm}$ of the circularly polarized QD ES PL, where $\tau_{\mathrm{CPD}}$ is the CPD decay time and $\tau_{\mathrm{PL}}^{\sigma \pm}$ are the PL decay times of the $\sigma^{ \pm}$polarizations, as a function of the QW thickness $t$. 
(a)
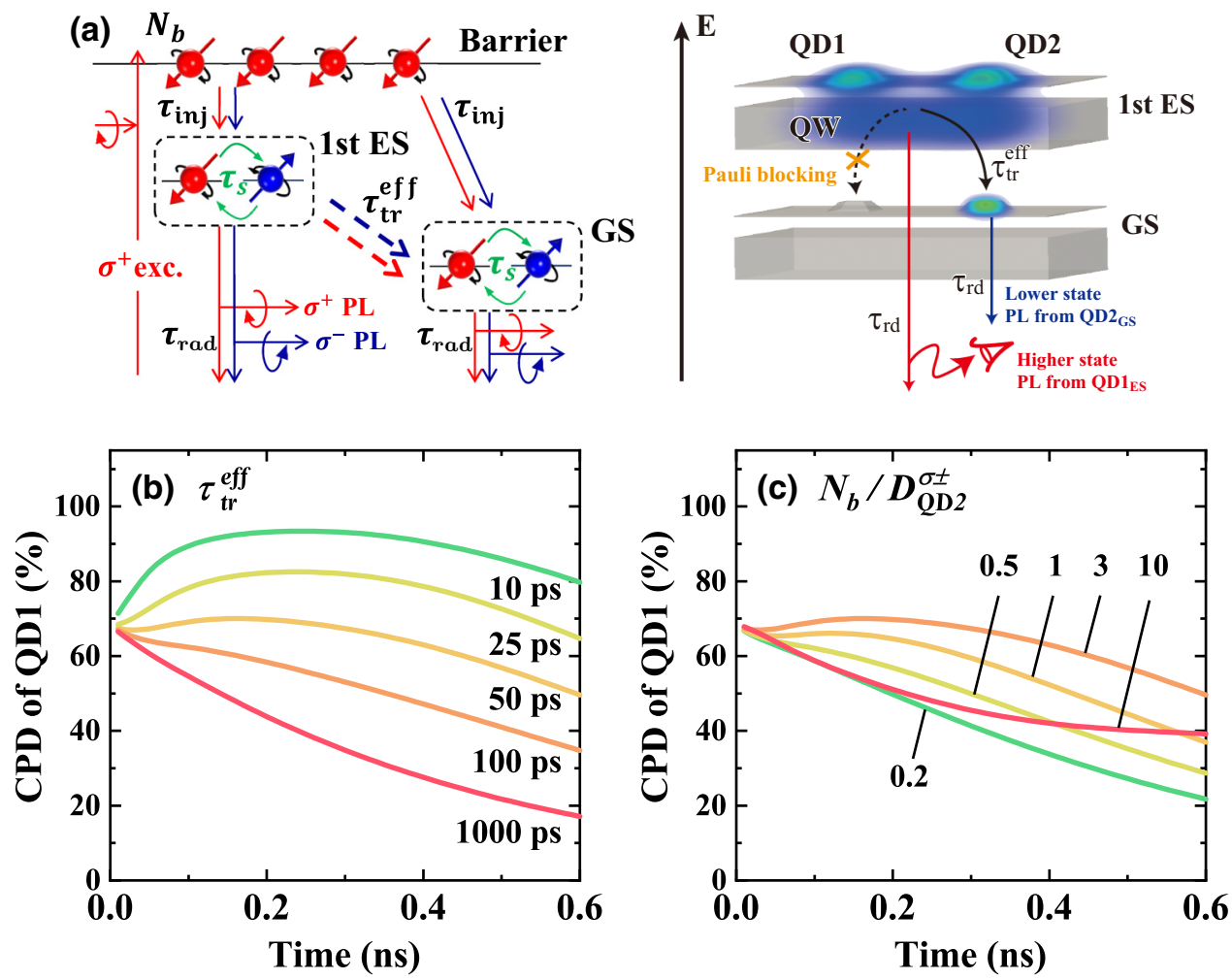

FIG. 4. (a) Spin-split rate equation model including electron-spin tunneling through coupling of electron wavefunctions in ESs and subsequent energy relaxation into a lowerenergy ground state in a single $\mathrm{QD}$, where the ESs detected by the time-resolved PL is expressed as first ES, while the ground state is expressed as GS (left-hand side). A schematic illustration of this model is also shown at the right-hand side. Simulation results for the transient CPD of the first ES for various (b) $\tau_{\text {tr }}^{\text {eff }}$ with a fixed parameter $N_{b} / D_{\mathrm{QD} 2}^{\sigma^{ \pm}}=3$, and (c) $N_{b} / D_{\text {QD2 }}^{\sigma^{ \pm}}$with $\tau_{\text {tr }}^{\text {eff }}=50$ ps.
The rate-equation model is illustrated in Fig. 4(a); the rate equations can be expressed as

$$
\begin{gathered}
\frac{d N_{b}}{d t}=-\frac{N_{b}}{\tau_{\mathrm{inj}}}\left\{\eta^{ \pm}\left(1-\frac{N_{\mathrm{QD} 1}^{\sigma^{ \pm}}}{D_{\mathrm{QD} 1}^{\sigma^{ \pm}}}\right)+\eta^{ \pm}\left(1-\frac{N_{\mathrm{QD} 2}^{\sigma^{ \pm}}}{D_{\mathrm{QD} 2}^{\sigma^{ \pm}}}\right)\right\}, \\
\frac{d N_{\mathrm{QD} 1(2)}^{\sigma \pm}}{d t}=\frac{N_{b}}{\tau_{\mathrm{inj}}} \eta^{ \pm}\left(1-\frac{N_{\mathrm{QD} 1(2)}^{\sigma \pm}}{D_{\mathrm{QD} 1(2)}^{\sigma \pm}}\right)+\frac{N_{\mathrm{QD} 1(2)}^{\sigma \mp}}{\tau_{s}} \\
-N_{\mathrm{QD} 1(2)}^{\sigma \pm}\left(\frac{1}{\tau_{s}}+\frac{1}{\tau_{\mathrm{rad}}}\right)-(+) \frac{N_{\mathrm{QD} 1}^{\sigma \pm}}{\tau_{\mathrm{tr}}^{\text {eff }}}\left(1-\frac{N_{\mathrm{QD} 2}^{\sigma \pm}}{D_{\mathrm{QD} 2}^{\sigma \pm}}\right),
\end{gathered}
$$

where $N_{b}$ and $\tau_{\text {inj }}$ are the initial population of a $\sigma^{+}$polarized (co-circular majority spin) electron at the barrier and time constant of spin injection from the barrier into a QD, respectively; $\eta^{ \pm}$denotes the spin-conservation factor during injection [31], and $\tau_{s}$ and $\tau_{\text {rad }}$ denote the time constants of spin relaxation and radiative recombination at the QD ES, respectively.

We introduce a parameter $\tau_{\text {tr }}^{\text {eff }}$, which is an effective time constant of the nonradiative decaying processes from the ESs, including thermal escape, trapping by defects or impurities, transformation into a dark exciton state, and energy relaxation to lower-energy sublevels in individual QDs, when electron wavefunctions are coupled in the ESs among neighboring QDs [labeled as first ES in Fig. 4(a)].
The final energy-relaxation processes should be dominant, as the thermal escape and trappings can be neglected at 6 $\mathrm{K}$ in the QDs. The transformation rate into the dark exciton is usually markedly slow and constant with respect to $t$ and excitation power. The strength of state filling is also included, as described in the above reference [23] using a parameter of $N_{b} / D$, where $D$ is the density of the spinsplit states. The two parameters of $\tau_{\mathrm{tr}}^{\text {eff }}$ and filling are of importance for the transient behavior of the CPD. Simulations of the transient CPD behavior as a function of time at the ESs (first ES) by varying the two parameters are shown in Figs. 4(b) and 4(c).The inherent values of $\tau_{\text {rad }}$ and $\tau_{s}$ at the ES are 0.3 and $0.8 \mathrm{~ns}$, respectively, obtained from separate experiments using QDs grown under identical conditions without QWs. A single exponential decay curve of the CPD is observed for $\tau_{\text {tr }}^{\text {eff }}=1 \mathrm{~ns}$ [Fig. 4(b)]; the CPD exhibits a nonmonotonic time response for a smaller $\tau_{\text {tr }}^{\text {eff }}$, though $\tau_{s}$ is fixed to $0.8 \mathrm{~ns}$. The time response of the CPD is almost constant at the initial time region, smaller than $0.3 \mathrm{~ns}$, with $\tau_{\mathrm{tr}}^{\text {eff }}=50 \mathrm{ps}$. The transient CPD exhibits an amplification at the time region smaller than $0.1 \mathrm{~ns}$ with a smaller $\tau_{\mathrm{tr}}^{\text {eff }}=10 \mathrm{ps}$. On the other hand, the state filling parameter $N_{b} / D$ for the lower state GS also affects the transient CPD behavior [Fig. 4(c)]. The value of 3 of this parameter reveals an almost time-insensitive CPD at the initial time region.

The excitation light powers are relatively strong to fill the excited states to which we pay attention. Auger effects are well known to occur in the QD systems and are 

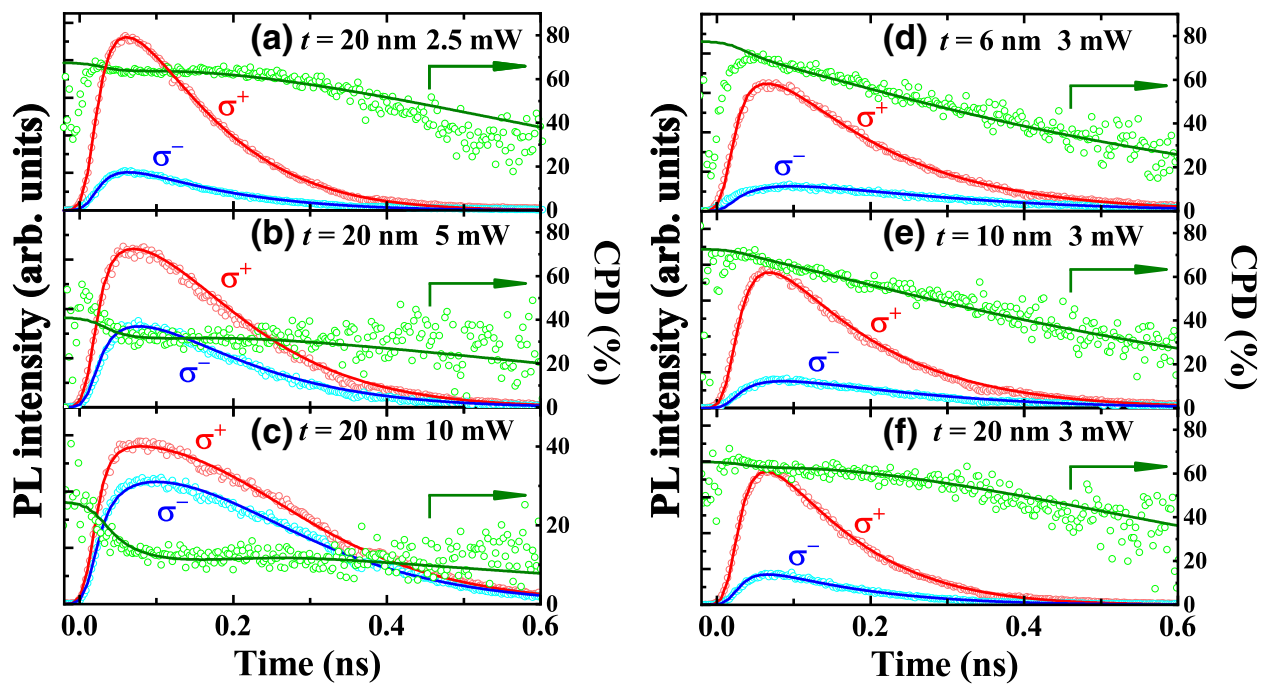

FIG. 5. Circularly polarized time-resolved PL and corresponding CPD, best fitted by rate equations (solid lines) for the samples with a 20-nm-thick QW at an excitation power of (a) 2.5, (b) 7.5, and (c) $10 \mathrm{~mW}$. The results at an excitation power of $3 \mathrm{~mW}$ for the samples with QW thicknesses $t$ of (d) 6 , (e) 10 , and (f) $20 \mathrm{~nm}$ are shown. observed in particular in wetting layers [32]. However, PL decay times of the majority spins are almost constant for the excitation power in all samples with different QW thicknesses. Therefore, the Auger scattering does not significantly affect the time response of the spin dynamics at the excited coupled states. This is reasonable since the QW thickness is much larger than that of a wetting layer and the density of the excited coupled states is also relatively large. In addition, we excite the layered GaAs barriers with the light penetration depth being several hundred $\mathrm{nm}$. The large number of photo-excited carriers or excitons diffuse toward the substrate since the coupled structure is grown at the relatively top side of the layered sample with the 40-nm-thick GaAs capping barrier. Slight influences of the Auger effect at those excited states can be additionally included in the above nonradiative decaying process.

These simulations demonstrate that moderate degrees of the state filling can lead to constant CPD values as a function of time in the light emission, combined with a proper $\tau_{\text {tr }}^{\text {eff }}$ value. The state filling of lower-energy majority-spin states in surrounding QDs can block the energy relaxation of the majority spins from the ES, as well as block the intradot relaxation into the GS. However, minority spins can relax to the above lower-energy states if those states are not fully occupied. Therefore, the total spin polarization is not degraded in the QD ensemble as the minority spins are removed from the ESs (first ES) detected by the time-resolved PL, i.e., transferred into the ground states (GS) in single dots, just after the flipping from the majority spin state. This mechanism clearly explains the previous difference in time-resolved PL decay time between the different polarizations, shown in Fig. 3(c). Detailed energy levels and the corresponding coupled states are explained in the Supplemental Material [33]. The temporal evolution of the circularly polarized PL at the ground states associated with the above transient circular polarizations at the excited states is also discussed in the Supplemental Material [33].

Figure 5 shows the least-square fittings of the rate equations for the circularly polarized time-resolved PL, with a convolution calculation of the instrumental time-response curve for the excitation laser pulse. The nonmonotonic and characteristic time responses of the CPD as well as circularly polarized transient PL intensities can be fully expressed. A combination of only two fitting parameters, $\tau_{\text {tr }}^{\text {eff }}$ and state filling, can explain all PL decay curves with both polarizations, and consequently the resultant CPD as a function of the time.

Figure 6 shows $\tau_{\text {tr }}^{\text {eff }}$ deduced from the fittings as a function of the excitation power for all samples. The value of $\tau_{\mathrm{tr}}^{\text {eff }}$ increases with the decrease of $t$, indicating a lower transfer rate, which can be attributed to the weaker coupling of the electron wavefunctions among QDs. In addition, $\tau_{\mathrm{tr}}^{\text {eff }}$ increases with the excitation power, as the

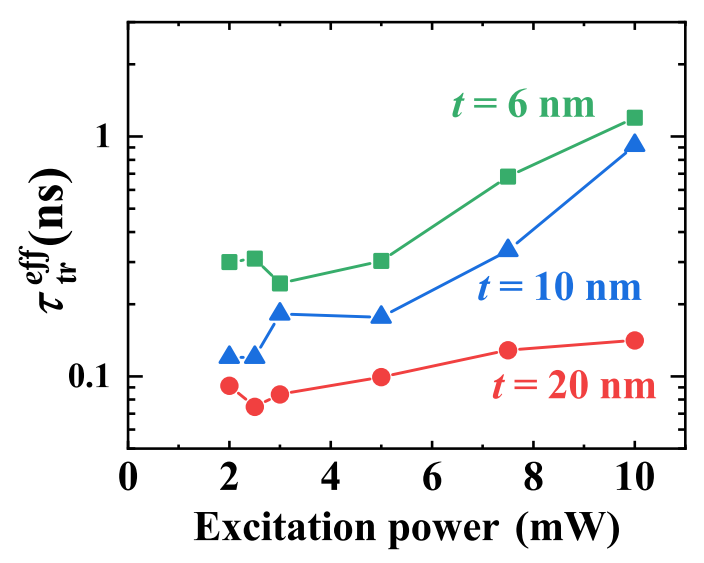

FIG. 6. Effective spin transfer time $\tau_{\text {tr }}^{\text {eff }}$ as a function of the excitation power for various $\mathrm{QW}$ thicknesses $t=6 \mathrm{~nm}$ (green squares), $10 \mathrm{~nm}$ (blue triangles), and $20 \mathrm{~nm}$ (red circles). 
state-filling effect at lower-energy minority spin states becomes more significant, which can prevent the transfer of minority spins among QDs. It should be noted that the state filling is an essential property for a sufficiently small QD with a discrete density of spin-split states. An enhanced tunneling of an electron spin among laterally aligned QDs is induced by the adjusted 2D QW potential; a thicker QW promotes an enhanced tunneling. The timeconstant PL CPD, i.e., persistent spin polarization during emissions at excited spin states in these QD ensembles with properly designed QWs is achieved by a combination of the above two parameters, an interdot spin transfer rate and state filling in particular for the minority spins.

\section{CONCLUSION}

Optical spin orientation and subsequent time-resolved PL spectroscopy with discriminating spin states are performed to study the spin dynamics at excited spinsplit states in unique QD-QW hybrid nanosystems of $\left(\mathrm{In}_{1-x} \mathrm{Ga}_{x}\right)$ As with various QW thicknesses. Rate equations considering the interdot spin transfer rate are used to explain the observed results aided by 3D calculations of the electron wavefunction. We observe an almost timeconstant and thus temporally persistent high spin polarization up to $70 \%$ during the PL emission at excited states of $\left(\operatorname{In}_{1-x} \mathrm{Ga}_{x}\right)$ As QDs laterally tunnel coupled through the 2D QW potential with a sufficient thickness. This temporally constant spin polarization can be sustained by a spatial transfer of minority spins among QDs, where the minority spins after the spin flip from the majority spins are selectively removed by the transfer process; therefore, the total spin polarization is maintained. This selective spin transfer is a function of the excited spin density, which can be attributed to a moderate state filling of lowerenergy spin sublevels in surrounding QDs. These findings show that the transient behavior of the electron-spin polarization, depending on the lateral coupling strength of the spin-polarized wavefunctions, can be intentionally and precisely controlled by the QW thickness of the designed QD-QW hybrid nanostructure. In addition, this suggests that these QD-QW hybrid nano-spin-systems are of significance for future developments of spin-functional optical devices. Furthermore, the concept of laterally coupled high-density QDs with properly designed QWs could be useful for various promising devices such as a highperformance QD laser [34] and QD-based memory [35]. It is also worth noting that solid-state quantum information processing requires a QD-based two-spin-level system with a precisely controlled coupling of the electron wavefunctions. Using the present coupled QD-QW structures, the interdot coupling can be controlled by applying an electric field normal to the layered structure. The potential shape of the QW along the thickness direction can be efficiently modified by the electric field, which can decouple the electron wavefunctions between QDs.

\section{ACKNOWLEDGMENTS}

This study was supported by the Japan Society for the Promotion of Science, Grant-in-Aid for Scientific Research S (KAKENHI No. 16H06359) and the bilateral program.

[1] Y. Arakawa and H. Sakaki, Multidimensional quantum well laser and temperature dependence of its threshold current, Appl. Phys. Lett. 40, 939 (1982).

[2] Y. Arakawa and A. Yariv, Quantum well lasers-Gain, spectra, dynamics, IEEE J. Quantum Electron QE-22, 1887 (1986).

[3] K. Mukai, Y. Nakata, H. Shoji, M. Sugawara, K. Ohtsubo, N. Yokoyama, and H. Ishikawa, Lasing with low threshold current and high output power from colummar-shaped InAs/GaAs quantum dots, Electron. Lett. 34, 1588 (1998).

[4] J. S. Wang, R. S. Hsiao, J. F. Chen, C. S. Yang, G. Lin, C. Y. Liang, C. M. Lai, H. Y. Liu, T. W. Chi, and J. Y. Chi, Engineering laser gain spectrum using electronic vertically coupled InAs-GaAs quantum dots, IEEE Photon. Technol. Lett. 17, 1590 (2005).

[5] K. Akahane, N. Yamamoto, and M. Tsuchiya, Highly stacked quantum-dot laser fabricated using a strain compensation technique, Appl. Phys. Lett. 93, 041121 (2008).

[6] A. Higo, T. Kiba, Y. Tamura, C. Thomas, J. Takayama, Y. Wang, H. Sodabanlu, M. Sugiyama, Y. Nakano, I. Yamashita, A. Murayama, and S. Samukawa, Lightemitting devices based on top-down fabricated GaAs quantum nanodisks, Sci. Rep. 5, 9371 (2015).

[7] A. Higo, T. Kiba, S. Chen, Y. Chen, T. Tanikawa, C. Thomas, C. Y. Lee, Y. C. Lai, T. Ozaki, J. Takayama, I. Yamashita, A. Murayama, and S. Samukawa, Optical study of sub-10 $\mathrm{nm} \mathrm{In}_{0.3} \mathrm{Ga}_{0.7} \mathrm{~N}$ quantum nanodisks in $\mathrm{GaN}$ nanopillars, ACS Photonics 4, 1851 (2017).

[8] A. V. Khaetskii and Y. V. Nazarov, Spin relaxation in semiconductor quantum dots, Phys. Rev. B 61, 12639 (2000).

[9] M. Paillard, X. Marie, P. Renucci, T. Amand, A. Jbeli, and J. M. Gérard, Spin Relaxation Quenching in Semiconductor Quantum Dots, Phys. Rev. Lett. 86, 1634 (2001).

[10] P. Borri, W. Langbein, S. Schneider, U. Woggon, R. L. Sellin, D. Ouyang, and D. Bimberg, Ultralong Dephasing Time in InGaAs Quantum Dots, Phys. Rev. Lett. 87, 157401 (2001).

[11] Y. Chye, M. E. White, E. Johnston-Halperin, B. D. Gerardot, D. D. Awschalom, and P. M. Petroff, Spin injection from $(\mathrm{Ga}, \mathrm{Mn}) \mathrm{As}$ into InAs quantum dots, Phys. Rev. B 66, 201301 (2002).

[12] C. H. Li, G. Kioseoglou, O. M. J. van 't Erve, M. E. Ware, D. Gammon, R. M. Stroud, and B. T. Jonker, Electrical spin pumping of quantum dots at room temperature, Appl. Phys. Lett. 86, 132503 (2005).

[13] L. Lombez, P. Renucci, P. F. Braun, H. Carrère, X. Marie, T. Amand, B. Urbaszek, and J. L. Gauffier, Electrical 
spin injection into p-doped quantum dots through a tunnel barrier, Appl. Phys. Lett. 90, 081111 (2007).

[14] D. Basu, D. Saha, C. C. Wu, M. Holub, Z. Mi, and P. Bhattacharya, Electrically injected InAs/GaAs quantum dot spin laser operating at $200 \mathrm{~K}$, Appl. Phys. Lett. 92, 091119 (2008).

[15] A. Tackeuchi, Y. Nakata, S. Muto, Y. Sugiyama, T. Usuki, Y. Nishikawa, N. Yokoyama, and O. Wada, Timeresolved study of carrier transfer among InAs/GaAs multicoupled quantum dots, Jpn. J. Appl. Phys. 34, L1439 (1995).

[16] Y. I. Mazur, J. W. Tomm, and V. Petrov, Staircase-like spectral dependence of ground-state luminescence time constants in high-density InAs/GaAs quantum dots, Appl. Phys. Lett. 78, 3214 (2001).

[17] R. Heitz, I. Mukhametzhanov, P. Chen, and A. Madhukar, Excitation transfer in self-organized asymmetric quantum dot pairs, Phys. Rev. B 58, R10151 (1998).

[18] A. Tackeuchi, T. Kuroda, K. Mase, Y. Nakata, and N. Yokoyama, Dynamics of carrier tunneling between vertically aligned double quantum dots, Phys. Rev. B 62, 1568 (2000).

[19] Y. I. Mazur, Z. M. Wang, G. G. Tarasov, G. J. Salamo, J. W. Tomm, V. Talalaev, and H. Kissel, Nonresonant tunneling carrier transfer in bilayer asymmetric InAs/GaAs quantum dots, Phys. Rev. B 71, 235313 (2005).

[20] X. J. Yang, T. Kiba, T. Yamamura, J. Takayama, A. Subagyo, K. Sueoka, and A. Murayama, Ultrafast spin tunneling and injection in coupled nanostructures of InGaAs quantum dots and quantum well, Appl. Phys. Lett. 104, 012406 (2014).

[21] S. L. Chen, T. Kiba, X. J. Yang, J. Takayama, and A. Murayama, Power-dependent spin amplification in (In, $\mathrm{Ga}) \mathrm{As} / \mathrm{GaAs}$ quantum well via Pauli blocking by tunnelcoupled quantum dot ensembles, Appl. Phys. Lett. 108, 152103 (2016).

[22] S. L. Chen, T. Kiba, X. J. Yang, J. Takayama, and A. Murayama, Temperature-dependent spin injection dynamics in InGaAs/GaAs quantum well-dot tunnel-coupled nanostructures, J. Appl. Phys. 119, 115701 (2016).

[23] T. Kiba, X.-j. Yang, T. Yamamura, Y. Kuno, A. Subagyo, K. Sueoka, and A. Murayama, Temperature dependence of the dynamics of optical spin injection in self-assembled InGaAs quantum dots, Appl. Phys. Lett. 103, 082405 (2013).

[24] T. Yamamura, T. Kiba, X. j. Yang, J. Takayama, A. Subagyo, K. Sueoka, and A. Murayama, Growth-temperature dependence of optical spin-injection dynamics in self- assembled InGaAs quantum dots, J. Appl. Phys. 116, 094309 (2014).

[25] T. Amano, T. Sugaya, and K. Komori, Characteristics of $1.3 \mu \mathrm{m}$ quantum-dot lasers with high-density and highuniformity quantum dots, Appl. Phys. Lett. 89, 171122 (2006).

[26] K. Watanabe, T. Akiyama, Y. Yokoyama, K. Takemasa, K. Nishia, Y. Tanaka, M. Sugawara, and Y. Arakawa, Growth of high-density $1.06-\mu \mathrm{m}$ InGaAs/GaAs quantum dots for high gain lasers by molecular beam epitaxy, J. Cryst. Growth 378, 627 (2013).

[27] F. Meier and B. P. Zakharchenya, Optical Orientation (North-Holland, Amsterdam, 1984).

[28] S. Birner, T. Zibold, T. Andlauer, T. Kubis, M. Sabathil, A. Trellakis, and P. Vogl, nextnano: General Purpose 3D Simulations, IEEE Trans. Electron. Devices 54, 2137 (2007).

[29] A. Tackeuchi, R. Ohtsubo, K. Yamaguchi, M. Murayama, T. Kitamura, T. Kuroda, and T. Takagahara, Spin relaxation dynamics in highly uniform InAs quantum dots, Appl. Phys. Lett. 84, 3576 (2004).

[30] V. K. Kalevich, M. Paillard, K. V. Kavokin, X. Marie, A. R. Kovsh, T. Amand, A. E. Zhukov, Y. G. Musikhin, V. M. Ustinov, E. Vanelle, and B. P. Zakharchenya, Spin redistribution due to Pauli blocking in quantum dots, Phys. Rev. B 64, 045309 (2001).

[31] D. Dagnelund, I. A. Buyanova, W. M. Chen, A. Murayama, T. Furuta, K. Hyomi, I. Souma, and Y. Oka, Efficiency of optical spin injection and spin loss from a diluted magnetic semiconductor $\mathrm{ZnMnSe}$ to $\mathrm{CdSe}$ nonmagnetic quantum dots, Phys. Rev. B 77, 035437 (2008).

[32] D. Morris, N. Perret, and S. Fafard, Carrier energy relaxation by means of Auger processes in InAs/GaAs selfassembled quantum dots, Appl. Phys. Lett. 75, 3593 (1999).

[33] See Supplemental Material at http://link.aps.org/supple mental/10.1103/PhysRevApplied.10.034015 for coupled excited states in the coupled QD-QW structures and the effects of multiple dot coupling, and time-evolution of circular polarization property at the QD ground state.

[34] W. Rudno-Rudziński, D. Biegańska, J. Misiewicz, F. Lelarge, B. Rousseau, and G. Sęk, Carrier diffusion as a measure of carrier/exciton transfer rate in InAs/InGaAsP/InP hybrid quantum dot-quantum well structures emitting at telecom spectral range, Appl. Phys. Lett. 112, 051103 (2018).

[35] A. Marent, T. Nowozin, M. Geller, and D. Bimberg, The QD-Flash: A quantum dot-based memory device, Semicond. Sci. Technol. 26, 014026 (2011). 